Research Paper

\title{
The impact of PRDX4 and the EGFR mutation status on cellular proliferation in lung adenocarcinoma
}

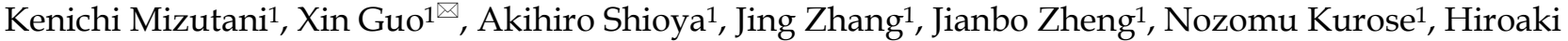 \\ Ishibashi², Nozomu Motono ${ }^{3}$, Hidetaka Uramoto ${ }^{3}$, Sohsuke Yamada ${ }^{1}$ \\ 1. Departments of Pathology and Laboratory Medicine, Kanazawa Medical University, Ishikawa, Japan. \\ 2. Departments of Oral and Maxillofacial Surgery, Kanazawa Medical University, Ishikawa, Japan \\ 3. Departments of Thoracic Surgery, Kanazawa Medical University, Ishikawa, Japan. \\ $\triangle$ Corresponding author: Xin Guo, M.D., Ph.D., Department of Pathology and Laboratory Medicine, Kanazawa Medical University, 1-1 Daigaku, Uchinada, \\ Kahoku, Ishikawa, 920-0293, Japan. Tel: 81-76-2188021; Fax: 81-76-286-1207; and E-mail: tianqi11211216@yahoo.co.jp. \\ (C) The author(s). This is an open access article distributed under the terms of the Creative Commons Attribution License (https://creativecommons.org/licenses/by/4.0/). \\ See http://ivyspring.com/terms for full terms and conditions.
}

Received: 2019.04.25; Accepted: 2019.07.10; Published: 2019.08.14

\begin{abstract}
Background: Oxidative stress plays key roles in the progression of lung adenocarcinoma. Recently, we reported that peroxiredoxin 4 (PRDX4), an antioxidant enzyme, can be a prognostic marker of lung adenocarcinoma (LUAD). In the present study, we aimed to further investigate the relationship among the PRDX4 expression, epidermal growth factor receptor (EGFR) mutations and cell proliferation in LUAD.

Methods: The expression of PRDX4 was immunohistochemically analyzed and the EGFR mutation status was examined in 127 paraffin-embedded human surgical specimens from patients with stage I LUAD. The PRDX 4 expression was considered to be high when $>40 \%$ of the adenocarcinoma cells were positively stained. In vitro, using plasmid transfection methods, PRDX4 plasmid DNAs were transfected into human lung adenocarcinoma cell lines, A549 (EGFR-wild) or PC-9 (EGFR mutant). The viability of these cells was analyzed using a Cell Counting Kit-8 kit.

Results: The number of cases with high PRDX4 expression levels among patients with LUAD with EGFR mutations was significantly larger than that in patients with EGFR wild-type. The combination of the PRDX4 expression level with the EGFR mutation status was closely associated with the prognosis of patients with stage I LUAD. Viability assays showed that the proliferation of A549 cells was significantly suppressed after PRDX4 plasmid transfection, while the overexpression of PRDX4 had no effect on the proliferation of EGFR-mutant PC-9 cells.

Conclusions: The PRDX4 expression and EGFR mutation status were significantly associated with the prognosis of patients with stage I LUAD, and EGFR mutations affected the role of PRDX4 in the proliferation of LUAD cells.
\end{abstract}

Key words: PRDX4; EGFR; cell proliferation; prognosis; lung adenocarcinoma (LUAD).

\section{Introduction}

Lung cancer has been one of the leading causes of cancer-related death in the world for two decades [1]. There were up to 370,000 cancer deaths and more than 74,000 lung cancer deaths in Japan in 2017. More than half of lung cancer cases are classified as non-small cell lung cancer (NSCLC); lung adenocarcinoma (LUAD) is a predominant subtype of these cases [2]. The 5-year overall survival rate is considered to be $<20 \%$ for NSCLC [3]. Amazingly, up to $30 \%$ of patients develop recurrent disease within 5 years, even those with stage I LUAD [4].

Oxidative stress can function as a crucial and diverse pathophysiological regulator of cellular signaling pathways, including growth factor stimulation [5], which can lead to the development of malignant neoplasms. In LUAD, the excessive expression of oxidative stressors plays a pivotal role in progression through cell signaling pathways 
closely related to tumor growth [6,7]. Peroxiredoxins (PRDXs), a new family of antioxidant enzymes, including at least six distinct PRDX genes expressed in mammals (PRDX1-6) [8], are ubiquitously synthesized and abundantly identified in various organisms. Recently, a large amount of clinical evidence indicates that PRDXs play important roles in the malignant progression of many cancers [9-13].

PRDX4, an antioxidant enzyme, is the only known secretory form of the PRDX family and is uniquely located in both the intracellular space and the extracellular space [14, 15]. In our previous series of studies, we showed that the overexpression of PRDX4 could prevent the progression of metabolic syndrome by reducing local and systemic oxidative stress and suppressing steatosis, inflammatory reactions, and/or apoptotic activity, suggesting that PRDX4 might be useful in the treatment of various chronic inflammatory diseases [16-19]. Recently, evidence demonstrated that different PRDX4 expression levels in tumor tissues were closely associated with the prognosis of cancer patients, indicating this antioxidant enzyme played important roles in the initiation and progression of cancer [20, 21]. In lung cancer, a report showed that high PRDX4 expression levels were significantly correlated with higher rates of recurrence and shorter disease-free survival (DFS) in patients with lung squamous cell carcinoma, but not in patients with lung adenocarcinoma [22]. Most recently, we reported that human LUAD tissues with low PRDX4 expression levels, a highly malignant phenotype that is very closely related to poor differentiation, highly invasive characteristics and recurrence, and the combination of low-PRDX4 and a high Ki-67 (MIB-1) labelling index may be a novel and useful independent predictor of recurrence with a poor prognosis in patients with primary stage I LUAD [23].

The epidermal growth factor receptor (EGFR), penetrating the cell membrane, is a member of the extensively studied receptor tyrosine kinase family. It can be activated by a family of ligands and induces downstream signaling pathways, which plays important roles in cell proliferation, differentiation, migration, and survival [24, 25]. Mutation of the EGFR gene leads to the aberrant activation of downstream signaling pathways and participates in critical mechanisms promoting tumorigenesis in malignant diseases, especially lung cancer [26, 27]. Pathologically, the aberrant expression of EGFR was found in more than half of non-small-cell lung cancer (NSCLC) patients [28] and mutations in the kinase domain of the EGFR gene are detected in approximately $40 \%$ of East Asian lung adenocarcinoma patients [29]. The EGFR mutation status has become one of the most important factors in the selection of lung cancer treatment, since EGFR Tyr kinase inhibitors (TKIs) have been widely used to treat NSCLC patients in recent years [30]. However, highly metastatic properties and drug resistance during TKI therapy-the precise mechanisms of which remain unclear-are still a problem to be solved for these NSCLC patients. It is well known that reactive oxygen species (ROS) are heavily involved in cancer initiation and regulation [31], and oxidative stress formation is an important mechanism participating in lung tumorigenesis through the regulation of the EGFR-mediated signaling pathway $[32,33]$.

One study reported that the intracellular overexpression of mouse PRDX4 prevented the production of ROS induced by epidermal growth factor (EGF) [34], indicating that EGFR may play an important role in the signaling pathways. Thus, there may be a close relationship between the expression of PRDX4 and EGFR mutation. However, there have been no studies on the relationships among the PRDX4 expression, the EGFR mutation status and cellular proliferation in LUAD. In this study, we examined the PRDX4 expression and EGFR mutation status in surgical specimens from patients with stage I LUAD. We also investigated the different roles of PRDX4 in the proliferation of EGFR wild-type and EGFR mutants in human LUAD cell lines in vitro.

\section{Materials and methods}

\section{Patients}

In the present study, we evaluated surgically resected stage I LUAD tissue specimens in which the EGFR mutation status had been evaluated. All specimens were obtained from patients who were treated at Kanazawa Medical University from January 2005 to December 2015. All materials in this article were approved by the Ethical Committee of Kanazawa Medical University (I159). Patients who suffered perioperative death, defined as death during the patient's initial hospitalization or within 30 days of surgery, were excluded. In addition, patients with the following characteristics were excluded: (a) other prior or concomitant malignant tumours, (b) coexisting medical problems of sufficient severity to shorten the life expectancy, and (c) adjuvant chemotherapies or radiotherapies prior to the surgery. After applying the exclusion criteria, a total of 127 patients with available follow-up data were included in this retrospective study.

Formalin-fixed, paraffin-embedded specimens were used for the IHC study. Clinical information was gathered from patient records. Patients were followed 
for 5 years after surgery. Disease-free survival (DFS) and disease-specific survival (DSS) were defined as the interval from the date of surgery to recurrence and from the date of surgery to death (except for patients who died from causes other than LUAD) or the most recent clinic visit, respectively.

\section{Histology and immunohistochemistry of tissue samples}

Three certified pathologists examined all resected specimens to evaluate their histopathological features. Tumors were classified according to the International Association for the Study of Lung Cancer (IASLC)/American Thoracic Society (ATS)/European Respiratory Society (ERS) classification [35]. A rabbit anti-PRDX4 IgG was produced and immunohistochemical staining was performed as previously described [23,36]. PRDX4 immunoreactivity was determined semi-quantitatively by evaluating the proportion of positively stained cells in comparison to the total number of adenocarcinoma cells.

\section{Cell culture}

A549, a LUAD cell line with wild-type EGFR, was obtained from the Department of Pathology, Kagoshima University. PC-9, a human LUAD cell line with EGFR mutation, was purchased from Riken BioResource Center (Tsukuba, Japan). These cells were cultured in Dulbecco's Modified Eagle Medium (DMEM) with $10 \%$ fetal calf serum and were maintained in a humidified atmosphere at $37^{\circ} \mathrm{C}$ under $95 \%$ air $/ 5 \% \mathrm{CO}_{2}$.

\section{Cell transfection}

PRDX4 plasmid DNA was obtained from the Department of Pathology, Kagoshima University. The cells were plated in 6-well plates and cultured in growth media at approximately 60\% confluence, incubated for $24 \mathrm{~h}$, and transfected for $72 \mathrm{~h}$ with $5 \mu \mathrm{g}$ PRDX4 plasmid DNA duplexes in Lipofectamine ${ }^{\circledR}$ 2000 and Opti-MEM medium (Life Technologies, Carlsbad, CA, USA). PMCV-Tag-2b (a gift from the Department of Pathology, Kagoshima University) vectors were used as a negative control respectively.

\section{Real-time PCR}

Total RNA from PC-9 and A549 cells was extracted using a Relia Prep RNA Cell Miniprep System (Promega) and was converted into cDNA using a High Capacity RNA-to-cDNA Kit (applied biosystems). The cDNA was analyzed using an Applied Biosytems QuantStudio 12K Flex Real-Time PCR system (Life Technologies) with TaqMan gene expression assays (Life Technologies). Each sample was analyzed in triplicate with separate wells for
PRDX4 and ribosomal 185 genes. The average values of three threshold cycle values for PRDX4 and 18S were calculated using the comparative $\mathrm{Ct}$ method. Custom-made primers and the TaqMan probe for PRDX4 gene amplification were purchased from Life Technologies (Assay ID: Hs01056076_m1).

\section{Western blotting}

Proteins isolated from A549 and PC-9 cells were separated by sodium dodecyl sulfate/polyacrylamide gel electrophoresis and transferred to PVDF Western Blotting Membranes (Roche Diagnostics $\mathrm{GmbH}$ ) using a semidry blotter. After transfer, the membranes were blocked with 5\% skim milk in TBST (TBS and $0.1 \%$ Tween 20 solution) for $1 \mathrm{~h}$ at room temperature (RT) and then incubated at RT with primary antibody diluted in TBST. The following primary antibodies and dilutions were used: PRDX4 Antibody (1:1000; Invitrogen) (rabbit polyclonal antibody), Anti $\beta$-Actin, MoAb, Peroxidase Conjugated (1:1000; Wako) (rabbit monoclonal antibody). After incubation with the primary antibody for PRDX4, the membrane was incubated with Anti-rabbit IgG, HRP-linked Antibody (1:1000; Cell Signaling) for $1 \mathrm{~h}$ at RT. The protein expression was detected with Clarity Western ECL Substrate (Bio-Rad).

\section{Cell proliferation assay}

The CCK-8 method was used to measure the viability of cells according to the manufacturer's instructions. A549 or PC-9 cells $\left(1 \times 10^{3}\right.$ cells) were seeded in one well of a 96-well plate and were observed for $72 \mathrm{~h}$. Absorbance at $450 \mathrm{~nm}$ was measured using a microplate reader.

\section{Statistical analyses}

All statistical analyses were performed using the $\mathrm{R}$ software program (version 3.2.3). The $\chi^{2}$ test or Fisher's exact test was used to assess relationships among the immunohistochemical expression of PRDX4, the EGFR mutation status and the clinicopathological features. Survival curves were analyzed using the Kaplan-Meier method and were compared using log-rank tests. The $t$-test was used to analyze continuous variables. All statistical analyses were two sided. $P$ values of $<0.05$ were considered to indicate statistical significance.

\section{Results}

\section{Patient characteristics}

The clinicopathological features of the 127 patients with stage I LUAD who were evaluated in the present study are summarized in Table 1 . The age of the patients at surgery range from 34 to 84 years (average, 68 years; median, 69 years). More than half 
of the patients $(80 / 127)$ had a Brinkman index (BI) of $<400$. The tumors were graded as follows: well-differentiated, $\mathrm{n}=72$; moderately differentiated, $\mathrm{n}=48$; and poorly differentiated, $\mathrm{n}=7$. The tumor size ranged from $6 \mathrm{~mm}$ to $50 \mathrm{~mm}$ (average, 22; median, 21 $\mathrm{mm})$. Pleural invasion, lymphatic invasion and venous invasion were found in 22, 46 and 43 cases, respectively.

Table 1. The clinicopathological characteristics of the patients

\begin{tabular}{ll}
\hline Characteristics & Patients $(\mathrm{n}=127)$ \\
\hline Age (years) & 68 \\
Average & 69 \\
Median & $34-84$ \\
Range & 106 \\
$>60$ & 21 \\
$\leq 60$ & \\
Sex & 63 \\
Male & 64 \\
Female & \\
Brinkman index (BI) & 47 \\
$\geqslant 400$ & 80 \\
$<400$ & \\
Tumor differentiation & 72 \\
Well & 48 \\
Moderate & 7 \\
Poor & \\
Tumor size (mm) & 22 \\
Average & 21 \\
Median & 63 \\
Range & \\
pl & \\
$(+)$ & 46 \\
$(-)$ & 22 \\
ly & 105 \\
$(+)$ & \\
$(-)$ & \\
v & \\
$(+)$ & \\
$(-)$ & \\
\hline
\end{tabular}

\section{The PRDX4 expression level and EGFR mutation status were correlated with the prognosis of patients with stage I LUAD}

To evaluate the relevance between the PRDX4
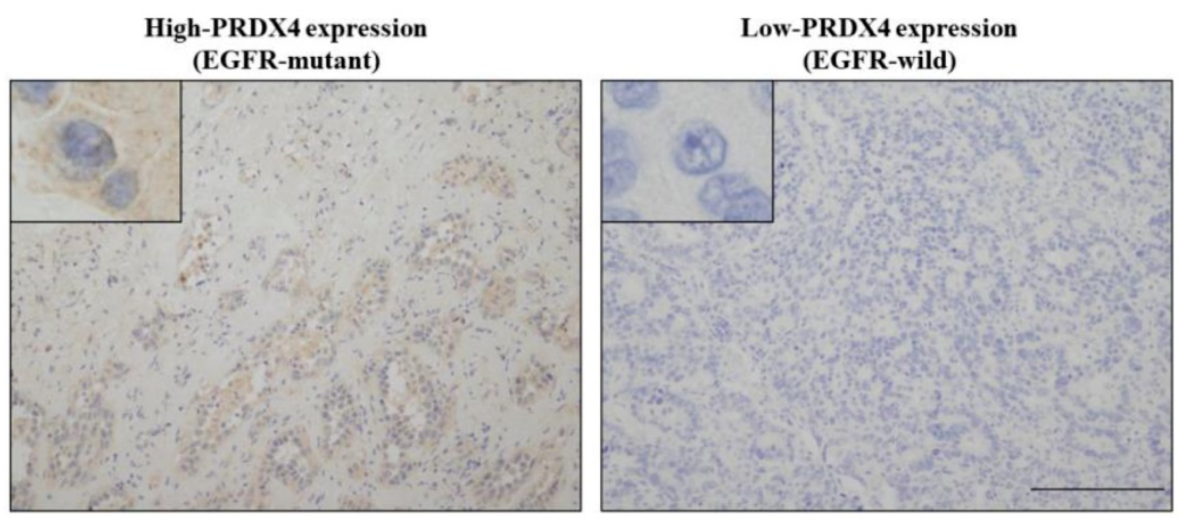

Figure 1. Representative images of the immunohistochemical analysis of PRDX4 (left, high-PRDX4; right, low-PRDX4) in patients with EGFR wild-type or EGFR mutations. The intracytoplasmic staining pattern of PRDX4 was confirmed. (Original magnification: $\times 100$; inset, $\times 400)$. Bar $=200 \mu \mathrm{m}(\times 100)$. 
A

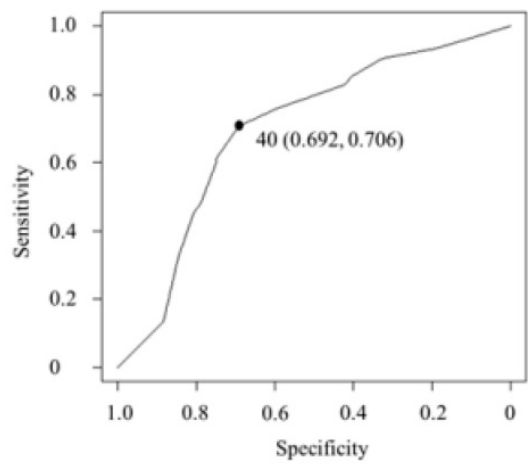

B

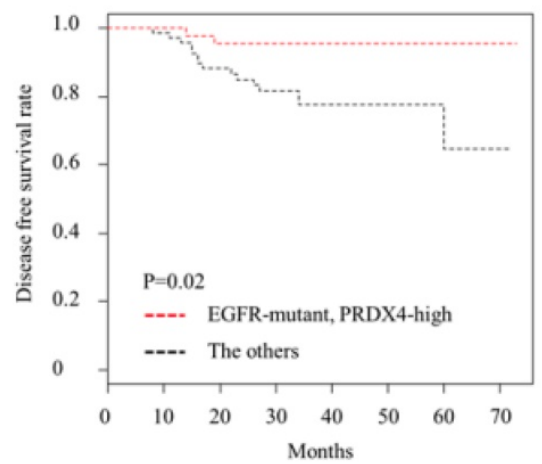

C

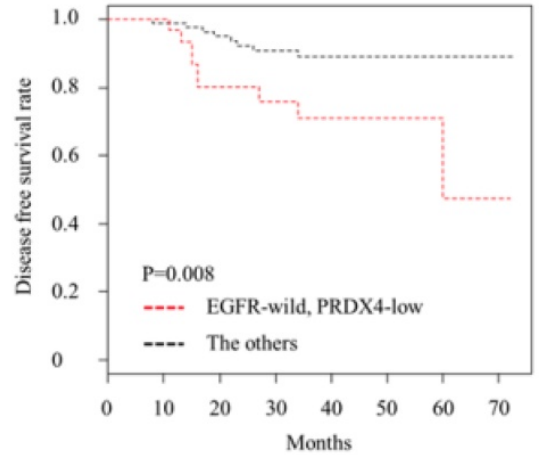

Figure 2. The receiver operating characteristic (ROC) curve analysis and Kaplan-Meier curves of the disease-free survival (DFS) in patients with Stage I LUAD after surgery according to the PRDX4 expression and the EGFR mutation status. (A) We selected $40 \%$ as the cut-off point for PRDX4, since the sum of sensitivity and specificity was the highest at this point. (B) Patients with EGFR mutations and high-PRDX4 showed significantly longer postsurgical DFS. (C) Patients with EGFR wild-type and low-PRDX4 showed significantly shorter postsurgical DFS.

A

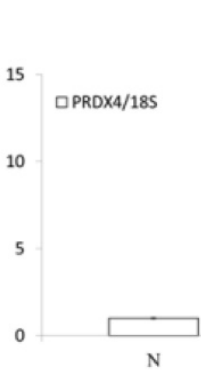

A549

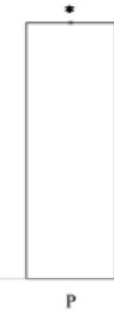

B

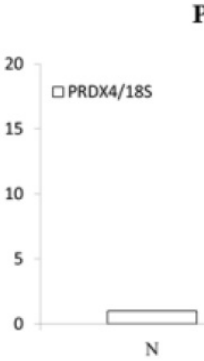

PC-9

C

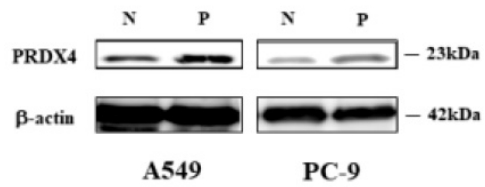

Figure 3. The results of the real-time PCR and Western blotting. The PRDX4 mRNA (A, B) and protein (C) expression was remarkably increased after transfection of PRDX4 plasmid in A549 and PC-9. N, negative control vector; P, PRDX4 plasmid.

A

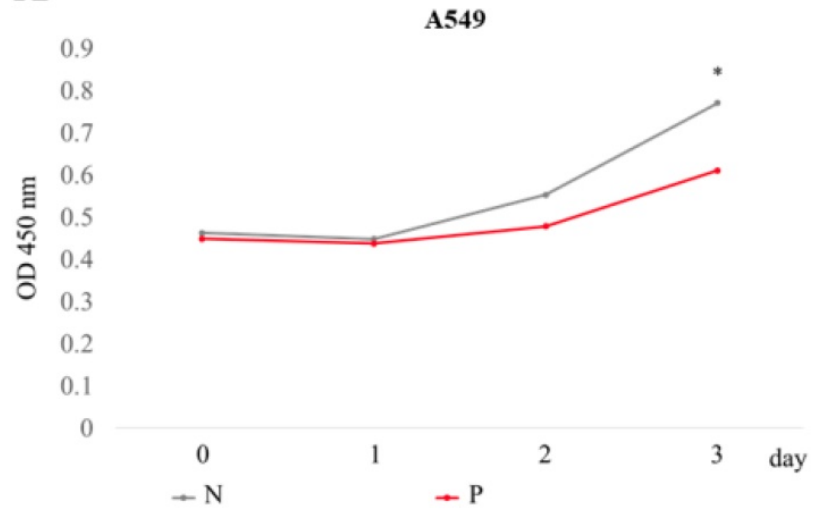

B

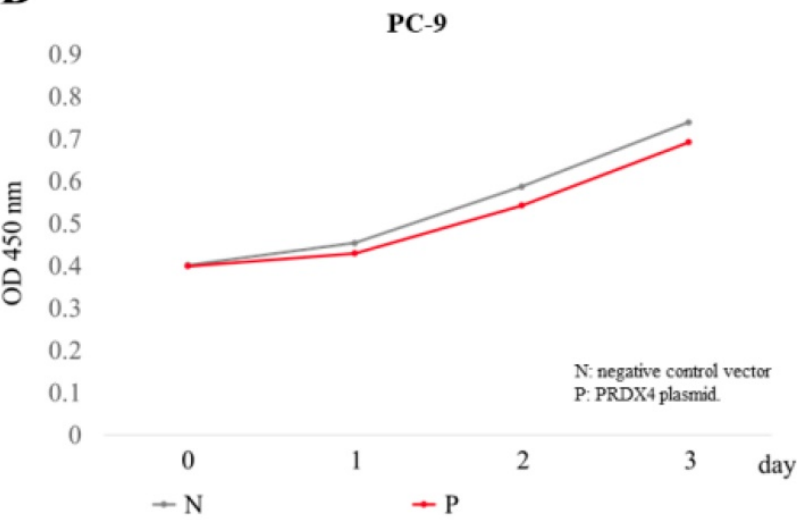

Figure 4. The overexpression of PRDX4 inhibited cell proliferation. The proliferation of A549 (A) and PC-9 (B) cells was analyzed using a cck-8 kit, 3 days after the transfection of PRDX4 plasmid DNAs or negative vectors. N, negative control vector; P, PRDX4 plasmid DNA. *p $<0.05$

\section{The overexpression of PRDX4 inhibited the proliferation of LUAD cells, but did not affect the LUAD cells with EGFR mutations}

Since it is well known that EGFR mutations usually lead to the abnormal expression of itself and promotes cellular proliferation in lung cancer, we investigated the roles of PRDX4 in the proliferation of two human LUAD cell lines, A549 cells (EGFR wild-type) and PC-9 cells (EGFR mutant). A significant increase in the expression of PRDX4
mRNA and protein was observed 3 days after transfection with PRDX4 plasmid DNA in both A549 and PC-9 cells (Fig. 3). Viability assays performed using a Cell Counting Kit-8 showed that the proliferation of A549 cells (EGFR wild-type) was significantly suppressed after the upregulation of the PRDX4 expression (Fig. 4A), but the overexpression of PRDX4 did not affect the proliferation of PC-9 cells (EGFR mutant) (Fig. 4B), indicating that EGFR mutation may attenuate the inhibitory role of PRDX4 in human LUAD cells. 


\section{Discussion}

The analysis of patients with stage I LUAD revealed that the EGFR mutant group included a larger number of patients with high PRDX4 expression levels than the EGFR wild-type group, indicating that there is a close relationship between the expression of PRDX4 and the EGFR mutation status. In addition, a Kaplan-Meier analysis showed that patients with EGFR mutations and high PRDX4 expression levels had significantly improved DFS in comparison to other patients, while the DFS of patients with EGFR wild-type and low PRDX4 expression levels was significantly reduced in comparison to other patients, suggesting that the combination of the PRDX4 expression and the EGFR mutation status may be an independent marker for postoperative recurrence in stage I LUAD patients.

EGFR belongs to the transmembrane receptor Tyr kinase family, which is triggered by ligand binding and the induction of EGFR homo-dimerization or hetero-dimerization [37] and regulates cell proliferation, migration, and survival [38]. The aberrant expression of the EGFR via gene amplification, mutation, or the overexpression of protein results in tumorigenesis, due to dysregulation of the EGFR-mediated signaling pathways, especially in lung cancer [39, 40]. Generally, the mutation of EGFR will lead to its overexpression [41], which could lead to excessive growth stimulation with the excess production of ROS [42]. Thus, in the present study, oxidative stress and an altered redox environment probably induced the expression of more antioxidants, including PRDX4, in order to promote cell survival. Several EGFR Tyr kinase inhibitors (TKIs) have been used as anticancer agents in the treatment of NSCLC in patients with EGFR mutations [43], and EGFR-TKIs show an positive therapeutic effect on EGFR-mutated NSCLC, recurrence and resistance to EGFR TKIs in LUAD patients after curative surgery remains a significant problem and limits the application of EGFR TKIs in NSCLC treatment [44], which leads to a poor prognosis in lung cancer patients. Thus, a better understanding of EGFR-regulated signaling pathways or other molecular mechanisms related to EGFR signaling is likely to have important clinical significance in cancer therapy for lung cancer patients. We summarized the hypothesized interactions between PRDX4 and EGFR in Supplementary Fig. 1.

In previous studies, we reported that tumor tissues of hepatocellular carcinoma and LUAD with high PRDX4 expression levels were significantly larger in size in comparison to those with low PRDX4 expression levels [21, 23], which was closely associated with the prognosis of these patients, suggesting that PRDX4 plays an important role in the proliferation of carcinoma cells. Indeed, in this study, our results showed that the overexpression of PRDX4 suppressed the rate of A549 cell proliferation, which was in line with the findings from our previous clinical study. Interestingly, however, the suppressive role of PRDX4 in cell proliferation was not observed in PC-9 cells (with EGFR mutation). The relatively higher ROS levels are suggested to be related to the proliferation of cancer cells [45]. Thus, it is easy to understand that the overexpression of PRDX4 may downregulate the intracellular ROS levels, which led to the inhibitory effects on the proliferation in A549 cells. However, EGFR-related molecular mechanisms may play more important roles in the processes of cell proliferation of PC-9 cells. At present, the precise mechanism underlying the inhibition of the suppressive roles of PRDX4 in the cellular proliferation of LUAD cells with EGFR mutations remains unclear. Further laboratory experiments are necessary to understand these findings.

The present study was associated with some limitations. Although we suggest that the number of human specimens was reasonable in this study, some important clinicopathological parameters related to DFS could not reach to statistical significance. Besides, all of the sample subjects resided relatively near to the single institution and had similar living habits with similar climatic conditions; thus, we could not avoid making a partial conclusion thoroughly. In addition, the in vitro analysis of cell proliferation only included one EGFR mutant cell line and the EGFR wild-type cell line.

\section{Conclusion}

In summary, the present study revealed-for the first time-a specific relationship between the expression PRDX4 and the EGFR mutation status, and further found that the combination of high-PRDX4 and EGFR mutation was closely associated with an improved prognosis whereas low-PRDX4 and EGFR wild-type was associated with worse DFS, suggesting that the combination of the PRDX4 expression and EGFR mutation status may be a novel prognostic biomarker for patients with stage I LUAD who have undergone surgery. Moreover, EGFR mutations may inhibit the suppressive role of PRDX4 in the proliferation of LUAD cells. Further laboratory experiments are needed to confirm the precise mechanism.

\section{Abbreviations}

LUAD: lung adenocarcinoma; PRDX4: peroxiredoxin 4; NSCLC: non-small cell lung cancer; TNM: tumor-node-metastasis; ROS: reactive oxygen 
species; DFS: disease-free survival; ROC: receiver operating characteristic.

\section{Supplementary Material}

Supplementary figures and tables. http://www.medsci.org/v16p1199s1.pdf

\section{Acknowledgments}

We would like to thank Yuka Hiramatsu, Mariko Nakano and Manabu Yamashita for their expert technical assistance.

\section{Funding}

This work was supported in part by Grants-in-Aid for Scientific Research 16K08750 to S.Y. and 17K10803 to H.U. from the Ministry of Education, Culture, Sports, Science and Technology, Tokyo, Japan; a grant from the MSD Life Science Foundation, Public Interest Incorporated Foundation, Japan (to S.Y.); and grants from National Natural Science Foundation of China (No. 81402490) (to X.G.), Natural Science Foundation of Hebei Province (No. H2016206170) (to X.G.) and High level talent support project of Hebei Province (No. CG2015003011) (to X.G.) and Grant for Promoted Research from Kanazawa Medical University (S2018-6) (to X.G.).

\section{Ethics approval}

All materials, including consent to participate in this article, were approved by the Ethical Committee of Kanazawa Medical University (I159).

\section{Consent for publication}

Written informed consent was obtained from the patient the patients and their family for the publication of this study and any accompanying images.

\section{Availability of data and materials}

The dataset supporting the findings and conclusions of this research is included within the article.

\section{Competing Interests}

The authors have declared that no competing interest exists.

\section{References}

1. Hidetaka U. Current Topics on Salvage Thoracic Surgery in Patients with Primary Lung Cancer. Annals of Thoracic and Cardiovascular Surgery. 2016;22(2):65-68

2. Lemjabbar-Alaoui $\mathrm{H}$, Hassan OU, Yang YW, Buchanan P. Lung cancer: biology and treatment options. Biochimica et Biophysica Acta. 2015;1856(2):189-210

3. Hidetaka U, Sohsuke Y, Fumihiro T. Angiogenesis of Lung Cancer Utilizes Existing Blood Vessels Rather than Developing New Vessels Using Signals from Carcinogenesis. Anticancer Research. 2013;33(5):1913-1916.

4. Taioli E, Yip R, Olkin I, Wolf A, Nicastri D, Henschke C, Yankelevitz D, Pass H Flores RM. Survival after Sublobar Resection for Early-Stage Lung Cancer:
Methodological Obstacles in Comparing the Efficacy to Lobectomy. Journal of Thoracic Oncology. 2016;11(3):400-406

5. Finkel T. Signal transduction by reactive oxygen species. The Journal of Cell Biology. 2011;194(1):7-15.

6. Okumura N, Yoshida H, Kitagishi Y, Nishimura Y, Iseki S, Matsuda S. Against Lung Cancer Cells: To Be, or Not to Be, That Is the Problem. Lung Cancer International. 2012;2012:659365.

7. Fruehauf JP1, Meyskens FL Jr. Reactive oxygen species: a breath of life or death? Clinical Cancer Research. 2007;13(3):789-94.

8. Rhee SG. Chae HZ. Kim K. Peroxiredoxins: A historical overview and speculative preview of novel mechanisms and emerging concepts in cell signaling. Free Radic Biol Med. 2005;38:1543-1552.

9. Ding C, Fan X, Wu G. Peroxiredoxin 1 - an antioxidant enzyme in cancer. J Cell Mol Med. 2017;21(1):193-202.

10. Zhang S, Fu Z, Wei J, Guo J, Liu M, Du K. Peroxiredoxin 2 is involved in vasculogenic mimicry formation by targeting VEGFR2 activation in colorectal cancer. Med Oncol. 2015;32(1):414.

11. Chang KP, Yu JS, Chien KY, Lee CW, Liang Y, Liao CT, Yen TC, Lee LY, Huang LL, Liu SC, Chang YS, Chi LM. Identification of PRDX4 and P4HA2 as metastasis-associated proteins in oral cavity squamous cell carcinoma by comparative tissue proteomics of microdissected specimens using ITRAQ technology. J Proteome Res. 2011;10(11):4935-47.

12. Seo MJ, Liu X, Chang M, Park JH. GATA-binding protein 1 is a novel transcription regulator of peroxiredoxin 5 in human breast cancer cells. Int J Oncol. 2012;40(3):655-64.

13. Quan C, Cha EJ, Lee HL, Han KH, Lee KM, Kim WJ. Enhanced expression of peroxiredoxin I and VI correlates with development, recurrence and progression of human bladder cancer. J Urol. 2006;175(4):1512-6.

14. Junichi F, Yoshitaka I, Toshihiro K, Takujiro H. Physiological and pathological views of peroxiredoxin 4. Free Radical Biology and Medicine. 2015;83:373-379.

15. Yamada S, Guo X Peroxiredoxin 4 (PRDX4): Its critical in vivo roles in animal models of metabolic syndrome ranging from atherosclerosis to nonalcoholic fatty liver disease. Pathology International. 2018;68(2):91-101.

16. Ding Y, Yamada S, Wang KY, Shimajiri S, Guo X, Tanimoto A, Murata Y, Kitajima S, Watanabe T, Izumi H, Kohno K, Sasaguri Y. Overexpression of peroxiredoxin 4 protects against high-dose streptozotocin-induced diabetes by suppressing oxidative stress and cytokines in transgenic mice. Antioxidant and Redox Signaling. 2010; 13(10): 1477-1490.

17. Guo $X$, Noguchi H, Ishii N, Homma T, Hamada T, Hiraki T, Zhang J, Matsuo K, Yokoyama S, Ishibashi H, Fukushige T, Kanekura T, Fujii J, Uramoto H, Tanimoto A, Yamada S. The Association of Peroxiredoxin 4 with the Initiation and Progression of Hepatocellular Carcinoma. Antioxidants and Redox Signaling. 2019;30(10):1271-1284.

18. Nabeshima A, Yamada S, Guo X, Tanimoto A, Wang KY, Shimajiri S, Kimura S, Tasaki T, Noguchi H, Kitada S, Watanabe T, Fujii J, Kohno K, Sasaguri Y. Peroxiredoxin 4 protects against nonalcoholic steatohepatitis and type 2 diabetes in a nongenetic mouse model. Antioxid Redox Signal. 2013;19(17):1983-98.

19. Nawata A, Noguchi H, Mazaki Y, Kurahashi T, Izumi H, Wang KY, Guo X, Uramoto H, Kohno K, Taniguchi H, Tanaka Y, Fujii J, Sasaguri Y, Tanimoto A, Nakayama T, Yamada S. Overexpression of peroxiredoxin 4 affects intestinal function in a dietary mouse model of nonalcoholic fatty liver disease. Public Library of Science One. 2016; 11(4): e0152549.

20. Ummanni R, Barreto F, Venz S, Scharf C, Barett C, Mannsperger HA, Brase JC, Kuner R, Schlomm T, Sauter G, Sültmann H, Korf U, Bokemeyer C, Walther R, Brümmendorf TH, Balabanov S. Peroxiredoxins 3 and 4 are overexpressed in prostate cancer tissue and affect the proliferation of prostate cancer cells in vitro. J Proteome Res. 2012;11(4):2452-66.

21. Guo X, Noguchi H, Ishii N, Homma T, Hamada T, Hiraki T, Zhang J, Matsuo K, Yokoyama S, Ishibashi H, Fukushige T, Kanekura T, Fujii J, Uramoto H, Tanimoto A, Yamada S. The Association of Peroxiredoxin 4 with the Initiation and Progression of Hepatocellular Carcinoma. Antioxidants and Redox Signaling. 2019;30(10):1271-1284.

22. Hwang JA, Song JS, Yu DY, Kim HR, Park HJ, Park YS, Kim WS, Choi CM. Peroxiredoxin 4 as an independent prognostic marker for survival in patients with early-stage lung squamous cell carcinoma. Int J Clin Exp Pathol. 2015;8(6):6627-35.

23. Shioya A, Guo X, Motono N, Mizuguchi S, Kurose N, Nakada S, Aikawa A, Ikeda Y, Uramoto H, Yamada S. The Combination Of Weak Expression Of PRDX4 And Very High MIB-1 Labelling Index Independently Predicts Shorter Disease-free Survival In Stage I Lung Adenocarcinoma. Int J Med Sci. 2018 Jun 14:15(10):1025-1034.

24. Wee P, Wang Z. Epidermal growth factor receptor cell proliferation signaling pathways. Cancers (Basel). 2017;9(5):E52.

25. Tomas A, Futter CE, Eden ER. EGF receptor trafficking: consequences for signaling and cancer. Trends Cell Biol. 2014;24(1):26-34.

26. Cooper WA, Lam DC, O'Toole SA, Minna JD. Molecular biology of lung cancer. J Thorac Dis. 2013;5(Suppl 5):S479-90.

27. Liu TC, Jin $X$, Wang $Y$, Wang $K$. Role of epidermal growth factor receptor in lung cancer and targeted therapies. Am J Cancer Res. 2017;7(2):187-202.

28. Gupta R, Dastane AM, Forozan F, Riley-Portuguez A, Chung F, Lopategui J, et al. Evaluation of EGFR abnormalities in patients with pulmonary adenocarcinoma: the need to test neoplasms with more than one method. Mod Pathol. 2009;22:128-33. 
29. Yatabe $Y$, Kerr KM, Utomo A et al. EGFR mutation testing practices within the Asia Pacific Region: results of a multicenter diagnostic survey. J Thorac Oncol 2015; 10: 438-45.

30. Lynch TJ, Bell DW, Sordella R, Gurubhagavatula S, Okimoto RA, Brannigan $\mathrm{BW}$, et al. Activating mutations in the epidermal growth factor receptor underlying responsiveness of non-small-cell lung cancer to gefitinib. N Engl J Med. 2004;350(21):2129-39.

31. Prasad S, Gupta SC, Tyagi AK. Reactive oxygen species (ROS) and cancer: role of antioxidative nutraceuticals. Cancer Lett. 2017;387:95-105.

32. Leung EL, Fan XX, Wong MP, Jiang ZH, Liu ZQ, Yao XJ, et al. Targeting tyrosine kinase inhibitor-resistant non-small cell lung Cancer by inducing epidermal growth factor receptor degradation via methionine 790 oxidation. Antioxid Redox Signal. 2016;24(5):263-79.

33. Lawless MW, O'Byrne KJ, Gray SG. Oxidative stress induced lung cancer and COPD: opportunities for epigenetic therapy. J Cell Mol Med. 2009;13(9A):2800-21.

34. Wong CM, Chun AC, Kok KH, Zhou Y, Fung PC, Kung HF, Jeang KT, Jin DY. Characterization of human and mouse peroxiredoxin IV: evidence for inhibition by Prx-IV of epidermal growth factor- and p53-induced reactive oxygen species. Antioxidants and Redox Signaling. 2000;2(3):507-18.

35. Travis WD, Brambilla E, Noguchi M, Nicholson AG, Geisinger KR, Yatabe $Y$, Beer DG, Powell CA, Riely GJ, Van Schil PE, Garg K, Austin JH, Asamura H, Rusch VW, Hirsch FR, Scagliotti G, Mitsudomi T, Huber RM, Ishikawa Y, Jett J, Sanchez-Cespedes M, Sculier JP, Takahashi T, Tsuboi M, Vansteenkiste I, Wistuba I, Yang PC, Aberle D, Brambilla C, Flieder D, Franklin W, Gazdar A, Gould M, Hasleton P, Henderson D, Johnson B, Johnson D, Kerr K, Kuriyama K, Lee JS, Miller VA, Petersen I, Roggli V, Rosell R, Saijo N, Thunnissen E, Tsao M, Yankelewitz D. International association for the study of lung cancer/american thoracic society/european respiratory society international multidisciplinary classification of lung adenocarcinoma. Journal of Thoracic Oncology. 2011;6(2):244-85

36. Ito R, Takahashi M, Ihara $\mathrm{H}$, Tsukamoto H, Fujii J, Ikeda $\mathrm{Y}$. Measurement of peroxiredoxin-4 serum levels in rat tissue and its use as a potential marker for hepatic disease. Molecular Medicine Reports. 2012;6(2):379-384.

37. Normanno N, De Luca A, Bianco C, Strizzi L, Mancino M, Maiello MR, et al. Epidermal growth factor receptor (EGFR) signaling in cancer. Gene. 2006;366(1):2-16.

38. Schlessinger J. Ligand-induced, receptor-mediated dimerization and activation of EGF receptor. Cell. 2002;110(6):669-72

39. Hirsch FR, Varella-Garcia M, Bunn PA Jr, Di Maria MV, Veve R, Bremmes RM, et al. Epidermal growth factor receptor in non-small-cell lung carcinomas: correlation between gene copy number and protein expression and impact on prognosis. J Clin Oncol. 2003;21(20):3798-807.

40. Kosaka T, Yatabe Y, Endoh H, Kuwano H, Takahashi T, Mitsudomi T. Mutations of the epidermal growth factor receptor gene in lung cancer: biological and clinical implications. Cancer Res. 2004;64(24):8919-23.

41. Salomon DS, Brandt R, Ciardiello F, Normanno N. Epidermal growth factor-related peptides and their receptors in human malignancies. Critical Reviews in Oncology/Hematology. 1995;19(3):183-232.

42. Prasad S, Gupta SC, Pandey MK, Tyagi AK, Deb L. Oxidative Stress and Cancer: Advances and Challenges. Oxid Med Cell Longev 2016: 5010423, 2016.

43. Maik-Rachline G, Seger R. The ERK cascade inhibitors: towards overcoming resistance. Drug Resist Updat. 2016;25:1-12.

44. Camidge DR, Pao W, Sequist LV. Acquired resistance to TKIs in solid tumours: learning from lung cancer. Nat Rev Clin Oncol. 2014;11(8):473-81.

45. Liou GY, Storz P. Reactive oxygen species in cancer. Free Radic Res 44: 479-496, 2010 